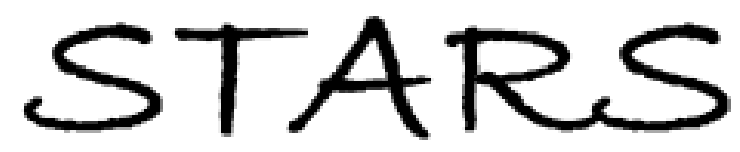

University of Central Florida

STARS

$1-1-1994$

\title{
Light-Scattering Observation Of Surface Acoustic Modes In High- Order Brillouin Zones Of A Si(001) Grating
}

\author{
Sukmock Lee \\ University of Central Florida \\ L. Giovannini \\ J. R. Dutcher \\ F. Nizzoli \\ G. I. Stegeman \\ University of Central Florida
}

See next page for additional authors

Find similar works at: https://stars.library.ucf.edu/facultybib1990

University of Central Florida Libraries http://library.ucf.edu

This Note is brought to you for free and open access by the Faculty Bibliography at STARS. It has been accepted for inclusion in Faculty Bibliography 1990s by an authorized administrator of STARS. For more information, please contactSTARS@ucf.edu.

\section{Recommended Citation}

Lee, Sukmock; Giovannini, L.; Dutcher, J. R.; Nizzoli, F.; Stegeman, G. I.; Marvin, A. M.; Wang, Z.; Ross, J. D.; Amoddeo, A.; and Caputi, L. S., "Light-Scattering Observation Of Surface Acoustic Modes In High-Order Brillouin Zones Of A Si(001) Grating" (1994). Faculty Bibliography 1990s. 3102.

https://stars.library.ucf.edu/facultybib1990/3102

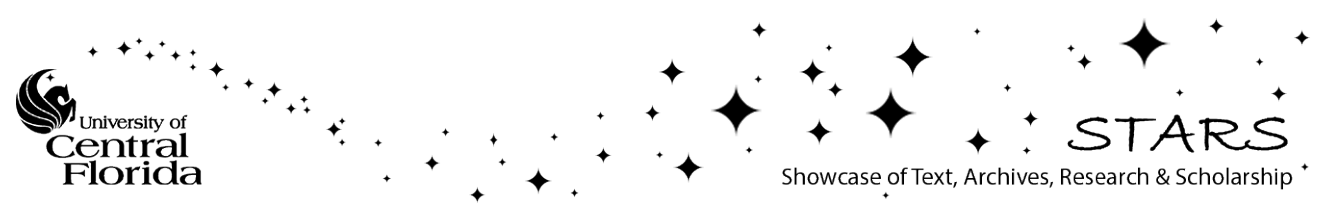




\section{Authors}

Sukmock Lee, L. Giovannini, J. R. Dutcher, F. Nizzoli, G. I. Stegeman, A. M. Marvin, Z. Wang, J. D. Ross, A. Amoddeo, and L. S. Caputi 


\title{
Light-scattering observation of surface acoustic modes in high-order Brillouin zones of a $\mathrm{Si}(001)$ grating
}

\author{
Sukmock Lee* \\ Center for Research and Education in Optics and Lasers, University of Central Florida, Orlando, Florida 32826 \\ L. Giovannini \\ Dipartimento di Fisica, Università di Ferrara, Via Paradiso 12, I-44100 Ferrara, Italy \\ J. R. Dutcher \\ Department of Physics, University of Guelph, Guelph, Ontario, Canada N1G 2W1 \\ F. Nizzoli \\ Dipartimento di Fisica, Università di Ferrara, Via Paradiso 12, I-44100 Ferrara, Italy \\ G. I. Stegeman \\ Center for Research and Education in Optics and Lasers, University of Central Florida, Orlando, Florida 32826
}

A. M. Marvin

Dipartimento di Fisica Teorica, Università di Trieste, Strada Costiera 11, I-34014 Miramare-Grignano, Italy

Z. Wang

Department of Physics, University of Guelph, Guelph, Ontario, Canada N1G 2W1

J. D. Ross

Center for Research and Education in Optics and Lasers, University of Central Florida, Orlando, Florida 32826

\author{
A. Amoddeo and L. S. Caputi \\ Dipartimento di Fisica, Università della Calabria, I-87036 Arcavacata di Rende (CS), Italy
}

(Received 11 October 1993)

\begin{abstract}
In Brillouin light-scattering (BLS) measurements of an ion-milled $\mathrm{Si}(001)$ surface grating with grating wavelength $\Lambda_{G}=0.35 \mu \mathrm{m}$, we have observed numerous high-order zone-folded surface acoustic modes between the second and third zone boundaries associated with the grating. A surprisingly intense signal from a zone-folded longitudinal resonance was observed in the absence of direct hybridization with the Rayleigh modes. The relative intensities of all of the modes have been calculated by allowing for coupling between modes related by up to two grating reciprocal-lattice vectors, and for a grating profile with nonzero first and second Fourier amplitudes. The Fourier amplitudes inferred from the BLS measurements and calculations agree very well with those obtained from a direct measurement of the grating profile using atomic-force microscopy.
\end{abstract}

Recently, Brillouin light-scattering (BLS) measurements of a shallow surface grating on a Si(001) wafer ${ }^{1}$ with grating wavelength $\Lambda_{G}=0.25 \mu \mathrm{m}$ have revealed a rich variety of grating-related features: zone folding of the Rayleigh mode, a gap in the Rayleigh mode dispersion for the phonon wave vector corresponding to the first grating zone boundary, and the unexpected observation of hybridization between the Rayleigh mode and the longitudinal resonance within the second grating zone. All of the grating-related features were explained quantitatively in a calculation of the normal modes and the BLS surface ripple cross section for a shallow grating on an opaque material. ${ }^{2}$ With the assumption of a cosinusoidal grating profile, excellent agreement between the measured and calculated BLS spectra was obtained both for modes with frequencies below and above the transverse threshold, corresponding to the discrete and continuous spectrum, respectively. ${ }^{3}$ This implies that the coupling between the discrete modes and the continuum occurs almost entirely via the fundamental periodicity of the grating, as specified by the first Fourier amplitude of the grating profile.

In this paper, we describe an extension of this original work to a larger period grating $\left(\Lambda_{G}=0.35 \mu \mathrm{m}\right)$, which allows us to measure spectra between the second and third grating zone boundaries. We have observed numerous zone-folded surface acoustic modes, including the observation of a zone-folded longitudinal resonance in the absence of direct hybridization with the Rayleigh modes. This latter observation is indeed unexpected because it is well known that the longitudinal resonance cannot be observed with BLS on the flat Si surface, ${ }^{4}$ and this mode has been observed for the $\Lambda_{G}=0.25 \mu \mathrm{m} \mathrm{Si}(001)$ grating only in the presence of a strong coupling with the Rayleigh 
mode. ${ }^{1,2}$ Our observations for the $\Lambda_{G}=0.35 \mu \mathrm{m}$ grating described in this paper are qualitatively different from those reported previously. The BLS calculation of Giovannini, Nizzoli, and Marvin ${ }^{2}$ has been extended to allow for coupling between modes with wave vectors related by up to two grating reciprocal-lattice vectors, and for a grating profile with nonzero first and second Fourier amplitudes. We find that the anomalously large scattering intensity for the zone-folded longitudinal resonance is confirmed by the calculation. In fact, excellent agreement is obtained between the experimental relative mode intensities for all of the modes observed in each of the BLS spectra and those calculated using the first and second Fourier amplitudes determined from BLS measurements of the first and second grating zone-boundary gaps. In addition, the Fourier amplitudes determined from the BLS data are in very good agreement with those obtained from a direct measurement of the grating profile using atomic-force microscopy.

The calculation of the normal modes of the grating and the ripple scattering cross section corresponding to each of these modes for a cosinusoidal grating profile has been described by Giovannini, Nizzoli, and Marvin. ${ }^{2}$ This calculation is valid for shallow gratings, since the Rayleigh hypothesis and a perturbative expansion to first order in the corrugation strength ${ }^{5}$ were used. To allow the proper description of all of the phonon modes with wave vectors between the second and third grating zone boundaries, this calculation has been extended to include the first two Fourier components: the grating profile $\zeta(x)$ is specified by

$$
\zeta(x)=2 \zeta_{G} \cos (G x)+2 \zeta_{2 G} \cos (2 G x),
$$

where $\zeta_{n G}$ are the Fourier amplitudes of the surface corrugation. Equation (1) can be used to describe symmetric grating profiles. In Eq. (1), the fundamental grating wave vector, or grating reciprocal-lattice vector $G$ is defined as $2 \pi / \Lambda_{G}$, such that the dispersion curves for the Rayleigh mode and longitudinal resonance are periodic in wave vector with period $G$. The first and second Fourier amplitudes $\zeta_{G}$ and $\zeta_{2 G}$, respectively, are primarily related to the measured frequency gaps of the Rayleigh mode at the first and second grating zone boundaries. ${ }^{5,6}$ In our theoretical approach, the first zone-boundary gap depends only on $\zeta_{G}$, while the second gap depends on both $\zeta_{2 G}$ (which gives the main contribution because of the mixing of two Rayleigh modes) and $\zeta_{G}$ (due to the mixing of the Rayleigh mode with the continuum). ${ }^{6}$ To calculate dispersion relations and spectra for wave vectors between the second and third grating zone boundaries, it is necessary, in principle, to include the effects due to the third Fourier amplitude $\zeta_{3 G}$. However, for the grating discussed below, we have found that the first two Fourier amplitudes give the only relevant contributions for the frequency and wave-vector ranges corresponding to the BLS data presented below. From a physical point of view, the calculation accounts for coupling between modes with wave vectors related by $\pm G$ and $\pm 2 G$. For the calculation of the BLS cross section, both the directscattering process ${ }^{2}$ and indirect-scattering process ${ }^{6}$ involving light diffraction through the first two Fourier am- plitudes of the corrugation have been taken into account.

The Si(001) grating, with the grating grooves parallel to the $\mathrm{Si}$ [100] direction, was prepared using the techniques described in Ref. 1 . The grating period was determined to be $\Lambda_{G}=0.349 \mu \mathrm{m}$ by accurate measurement of the first-order-diffracted beam deflection. The BLS experiments were performed at room temperature using a Sandercock-type high contrast, tandem $(3+3$ passes $)$ Fabry-Pérot interferometer. ${ }^{7} 100 \mathrm{~mW}$ of $p$-polarized light from a single mode $\mathrm{Ar}^{+}$laser $(\lambda=5145 \AA)$ was focused onto the grating surface using a $f / 1.4(f=50 \mathrm{~mm})$ lens. Diffusely scattered $p$ - and $s$-polarized light was collected by the same lens using a $180^{\circ}$ backscattering geometry. A narrow slit was placed in the collected beam, offset slightly from the beam center, to avoid artificial splitting of the BLS peaks. ${ }^{1}$ The plane of incidence of the light was along the [010] direction of the $\mathrm{Si}(001)$ crystal, perpendicular to the grating grooves.

A BLS spectrum collected for a phonon wave-vector value of $Q_{\|}=2.373 \times 10^{5} \mathrm{~cm}^{-1}$ (angle of incidence $\theta_{i}=78^{\circ}$ and scattering angle $\theta_{s}=75^{\circ}$ ) is shown in Fig. 1(a). The large peak at $18 \mathrm{GHz}$ corresponds to light scattering from the Rayleigh mode that would be observed for a flat surface. In addition, there are smaller peaks at 4.6, 7.9, and 9.2 GHz, which correspond to light scattering from modes which exist and are observed because of the presence of the surface grating. Because of its very low frequency, the peak at $4.6 \mathrm{GHz}$ sits on the edge of the background due to the very large elasticscattering peak at $0 \mathrm{GHz}$. The character of each of these modes can be understood from a plot of the frequencydispersion curves.

In Fig. 2 are shown the frequency-dispersion curves for

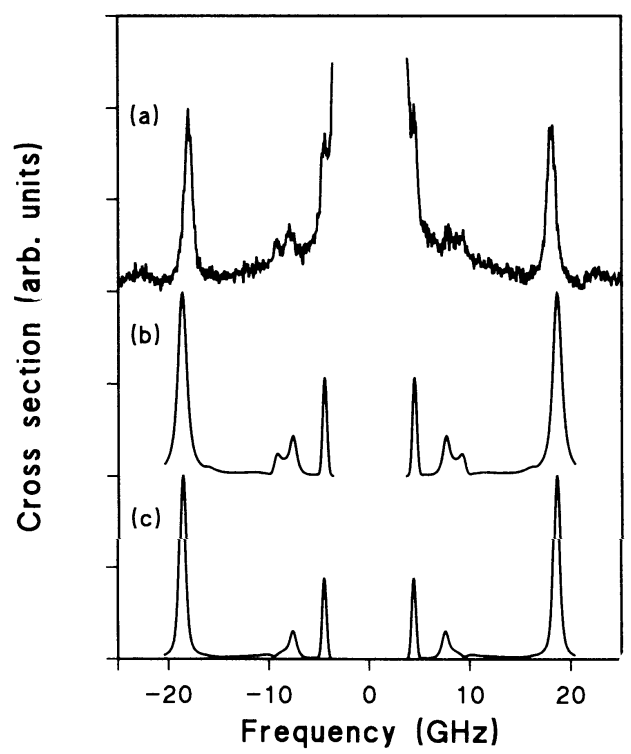

FIG. 1. Brillouin light-scattering spectra for the $\mathrm{Si}(001)$ grating with $\Lambda_{G}=0.35 \mu \mathrm{m}$, for a phonon wave vector of $2.373 \times 10^{5}$ $\mathrm{cm}^{-1}$. (a) Experimental spectrum, collected using $100 \mathrm{~mW}$ of laser power, and $50.9 \mathrm{~s}$ of counting time per data point; (b) spectrum calculated using Fourier amplitudes of $\zeta_{G}=125 \AA$ and $\xi_{2 G}=40 \AA$; and (c) spectrum calculated using $\zeta_{G}=125 \AA$ and $\xi_{2 G}=0$. 


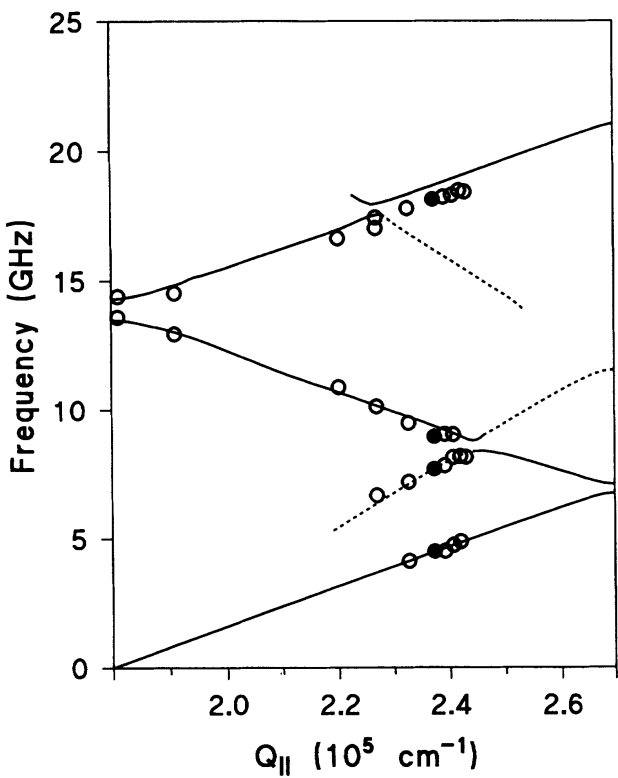

FIG. 2. Frequency-dispersion curves for the $\mathrm{Si}(001)$ grating with $\Lambda_{G}=0.35 \mu \mathrm{m}$ between the second and third grating zone boundaries. The open and solid circles correspond to data, with the solid circles corresponding to the BLS spectra shown in 1(a). The solid and dashed curves represent the dispersion of the zone-folded Rayleigh mode and longitudinal resonance, respectively, calculated using the theory described in the text.

the Rayleigh mode and the longitudinal resonance of the $\mathrm{Si}(001)$ grating for phonon wave vectors between the second and third grating zone boundaries. The data corresponding to the BLS spectrum of Fig. 1(a) are shown as solid circles. The curves correspond to the periodic zone scheme representation of the Rayleigh mode and longitudinal resonance. Data and curves are limited to the third grating zone which corresponds to the actual phonon wave vectors probed in BLS experiments. These curves have been calculated using the first and second Fourier amplitudes $\zeta_{G}=125 \AA$ and $\zeta_{2 G}=40 \AA$ that gave the best fit to BLS measurements of the first and second zoneboundary gaps $\Delta f_{1}=0.4 \mathrm{GHz}$ and $\Delta f_{2}=0.8 \mathrm{GHz}$, respectively. These Fourier amplitudes have been fitted also by considering the behavior of the peak intensities near the grating zone-boundary gaps. Actually, for wave vectors close to the zone boundaries, the mode intensities depend strongly on $Q_{\|}$, i.e., on the incident and scattering angles $\theta_{i}$ and $\theta_{s}$. The intrinsic experimental uncertainty in the determination of $\theta_{s}$ of $\pm 0.25^{\circ}$ produces a typical uncertainty in the determination of $\Delta f_{1}$ and $\Delta f_{2}$ of $\pm 15 \%$. We estimate that our determinations of $\zeta_{G}$ and $\zeta_{2 G}$ are described by the same $\pm 15 \%$ uncertainty. ${ }^{6}$ The measurement of $\Delta f_{2}$ at the second grating zone boundary was complicated by the presence in the collected scattered light of an intense beam due to first-order diffraction from the grating. We found that it was possible to block this small diameter $(3 \mathrm{~mm})$ beam with a small mask inserted into the collected scattered light. For this grating, the depth is determined primarily by $\zeta_{G}$; the corrugation strength $\left(2 \zeta_{G}\right) / \Lambda_{G}=0.07$ corresponds to the small roughness limit, as for the grating discussed in
Refs. 1 and 2.

In Fig. 2, the highest frequency branch of the dispersion curves corresponds to the Rayleigh mode branch that would be observed for a flat $\mathrm{Si}(001)$ surface (flatsurface-like Rayleigh mode). The lowest frequency branch corresponds to the Rayleigh mode branch which has been shifted in wave vector by $+G(+G$ Rayleigh mode). The intermediate frequency branch with negative slope corresponds to the Rayleigh mode branch which has been shifted in wave vector by $+2 G(+2 G$ Rayleigh mode). The intermediate frequency branch with positive slope corresponds to the longitudinal resonance branch which has been shifted in wave vector by $+G(+G$ longitudinal resonance). Therefore, for the BLS spectrum shown in Fig. 1(a), the peaks correspond to, in order of increasing frequency, a $+G$ Rayleigh mode, a $+G$ longitudinal resonance, a $+2 G$ Rayleigh mode, and the flatsurface-like Rayleigh mode. Associated with each Rayleigh mode branch is a transverse threshold which has a frequency that is about $10 \%$ larger than that of the Rayleigh mode. For mode frequencies above the lowest transverse threshold, the modes are no longer discrete and are called "leaky" modes ${ }^{3}$ with respect to the continuum, since they can decay into bulk modes. Therefore, in the spectrum of Fig. 1(a), only the lowest frequency $+G$ Rayleigh mode is discrete. The $+G$ longitudinal resonance and the $+2 G$ Rayleigh mode are both leaky with respect to the $+G$ continuum, and the flat-surface-like Rayleigh mode is leaky with respect to both the $+G$ and the $+2 G$ continua. Note that the dispersion relations of the leaky modes have been taken from the corresponding maxima in the calculated BLS spectra.

As can be seen in Fig. 2, the $+G$ longitudinal resonance is observed for wave vectors up to $7 \%$ less than the wave vector corresponding to the gap between the $+G$ longitudinal resonance and the $+2 G$ Rayleigh mode, with differences between the two mode frequencies of up to $3.5 \mathrm{GHz}$. This is in dramatic contrast to the observations of Dutcher et al., ${ }^{1}$ in which the $+G$ longitudinal resonance was observed only for wave vectors within $\pm 3 \%$ of the wave vector corresponding to the largest mixing of the $+G$ longitudinal resonance and the flatsurface-like Rayleigh mode, with differences between the two mode frequencies of less than $1 \mathrm{GHz}$.

In Fig. 1(b) a spectrum is shown calculated using the Fourier amplitudes given above. All of the peaks observed in the BLS experiment also appear in the calculated spectrum, and the agreement between the experimentally observed and calculated relative scattering intensities of the modes is excellent. The effect of including the second Fourier component can be seen by comparing the calculated spectrum in Fig. 1(b) to the calculated spectrum in Fig. 1(c), for which the second Fourier amplitude has been set equal to zero. Not surprisingly, the relative intensity of the Rayleigh mode near $9 \mathrm{GHz}$ that is shifted in wave vector by $+2 G$ is very sensitive to the second Fourier amplitude. Note that with $\xi_{2 G}=0$, the $+2 G$ Rayleigh mode is marked by the presence of a dip instead of a peak. This is due to destructive interference effects in the surface phonon power spectrum occurring through modes coupled by $\zeta_{G}^{2}$. Also, the linewidth of the flat- 
surface-like Rayleigh mode increases with increasing $\zeta_{2 G}$, due to the increase in the "leakiness" of the mode with respect to the $+2 G$ continuum. This effect, together with the $1 / \Omega^{2}$ dependence of the cross section, ${ }^{2}$ contributes to a decrease in the intensity of this mode with respect to the intensities of the low-frequency modes.

The grating profile was measured directly using atomic-force microscopy (AFM). The AFM measurements were carried out in air using a Nanoscope III (Digital Instruments) operating in the constant force mode. It combines a commercial $\mathrm{Si}_{3} \mathrm{~N}_{4}$ cantilever with the optical lever technique. The AFM microscope was equipped with standard Nanoprobe tips with a typical end radius of curvature of about $300 \AA$. A square area of the grating was scanned that was $1.358 \mu \mathrm{m}$ on a side. Data were acquired automatically using a digital processor connected to a personal computer and it was stored as digitized images with a $256 \times 256$ grid. Several periods of the grating can be seen in the atomic-force micrograph shown in Fig. 3. A single AFM line scan perpendicular to the grating grooves is shown as an inset to Fig. 3, together with the grating profile calculated with first and second Fourier amplitudes $\zeta_{G}=135 \pm 5 \AA$ and $\zeta_{2 G}=60 \pm 5 \AA$ that give the best agreement with the measured AFM profile. The error estimates apply to a fit performed using the single line scan of the AFM image. The AFM profile is well reproduced by the calculated profile, except for a small asymmetry present near the bottom of the grating grooves which cannot be described by the profile defined by Eq. (1). The agreement of the $\zeta_{G}$ and $\zeta_{2 G}$ values determined independently from the BLS and AFM data is remarkable, considering the approximation involved in reproducing the details of the true grating profile with the simple expansion given by Eq. (1), and it gives us confidence that we understand the BLS properties of the $\mathrm{Si}(001)$ grating.

In summary, we have observed up to four different surface modes in a single BLS spectrum collected for a $\mathrm{Si}(001)$ grating. The unexpected observation of a zone-

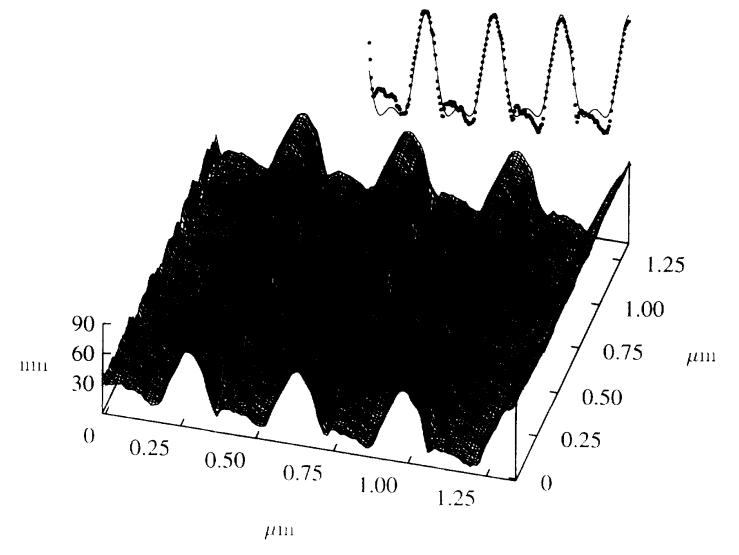

FIG. 3. Atomic-force microscope (AFM) image of the $\mathrm{Si}(001)$ grating surface. In the inset to the figure is shown a comparison between one of the AFM line scans perpendicular to the grating grooves and the grating profile calculated using Eq. (1) and the best-fit Fourier amplitudes determined from the AFM data: $\zeta_{G}=135 \AA$ and $\zeta_{2 G}=60 \AA$.

folded longitudinal resonance in the absence of direct hybridization with the Rayleigh modes is reproduced in calculated BLS spectra. We find excellent agreement between the measured and calculated mode dispersion and relative mode intensities. In addition, very good agreement is obtained between the set of first and second Fourier amplitudes obtained independently from the BLS and AFM data.

The authors gratefully acknowledge financial support from the Natural Sciences and Engineering Research Council (NSERC) of Canada (J.R.D. and S.L.), the Ministero Università e Ricerca Scientifica e Tecnologica (L.G., F.N., A.M.M., A.A., and L.S.C.), and the Basic Science Research Institute Program of Korea (S.L.).
*Present address: Department of Physics, Inha University, Inchon 402-751, Korea.

${ }^{1}$ J. R. Dutcher, S. Lee, B. Hillebrands, G. J. McLaughlin, B. G. Nickel, and G. I. Stegeman, Phys. Rev. Lett. 68, 2464 (1992).

${ }^{2}$ L. Giovannini, F. Nizzoli, and A. M. Marvin, Phys. Rev. Lett. 69, 1572 (1992).

${ }^{3}$ V. Bortolani, F. Nizzoli, and G. Santoro, Phys. Rev. Lett. 41, 39 (1978).
${ }^{4} J$. R. Sandercock, Solid State Commun. 26, 547 (1978),

${ }^{5}$ N. E. Glass, R. Loudon, and A. A. Maradudin, Phys. Rev. B 24, 6843 (1981).

${ }^{6}$ L. Giovannini, F. Nizzoli, and A. M. Marvin, J. Electron. Spectrosc. Relat. Phenom. (to be published).

${ }^{7}$ J. R. Sandercock, in Light Scattering in Solids III, edited by M. Cardona and G. Güntherodt (Springer-Verlag, Berlin, 1982), p. 173. 


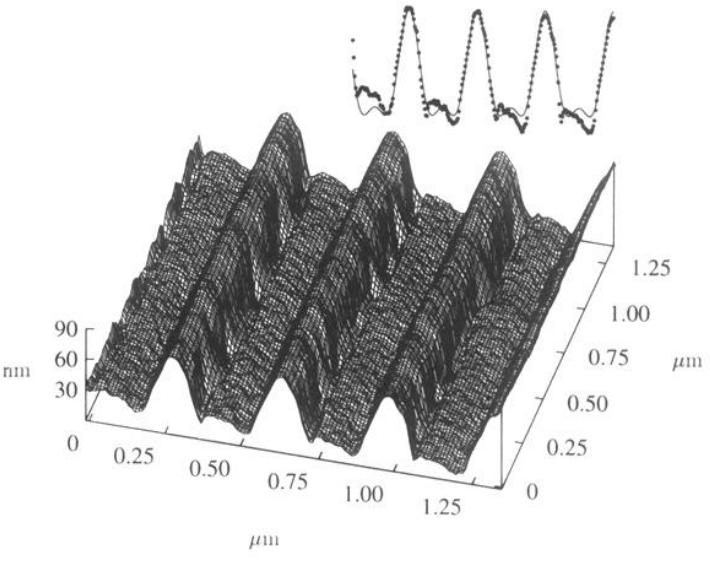

FIG. 3. Atomic-force microscope (AFM) image of the Si(001) grating surface. In the inset to the figure is shown a comparison between one of the AFM line scans perpendicular to the grating grooves and the grating profile calculated using Eq. (1) and the best-fit Fourier amplitudes determined from the AFM data: $\zeta_{G}=135 \AA$ and $\zeta_{2 G}=60 \AA$. 